\title{
Lesión de aparato abductor posterior a artroplastia total de cadera tratado con transferencia muscular de glúteo mayor a trocánter mayor
}

\author{
Injury to the hip abductors after total hip arthroplasty treated with \\ muscle transfer from gluteus maximus to the greater trochanter
}

\author{
Jorge Arturo Barraza-Silva, ${ }^{*}$ Rodrigo Sánchez Rowold, ${ }^{\ddagger}$ Roberto Pareyón Valero, ${ }^{\ddagger}$ Javier Camacho Galindo§
}

Citar como: Barraza-Silva JA, Sánchez RR, Pareyón VR, Camacho GJ. Lesión de aparato abductor posterior a artroplastia total de cadera tratado con transferencia muscular de glúteo mayor a trocánter mayor. An Med ABC. 2021; 66 (2): 137-141. https://dx.doi. org/10.35366/100484

\section{RESUMEN}

La lesión del aparato abductor de la cadera es una entidad clínica bien caracterizada que condiciona dolor sobre el trocánter mayor, y es acompañado de debilidad y claudicación progresivas. Estas lesiones pueden ocurrir en casos que fueron sometidos a artroplastia total de cadera o en caderas nativas. El tratamiento en ambas situaciones depende de la localización de la lesión y el grado de infiltración de grasa muscular. La transferencia descrita por Whiteside del glúteo máximo al trocánter mayor otorga adecuados resultados para el control del dolor y aumento de fuerza del mecanismo abductor. En este caso clínico, se presenta una paciente sometida a una artroplastia total de cadera izquierda que desarrolló una rotura completa tendinosa del glúteo medio, así como el abordaje diagnóstico y terapéutico de esta lesión.

Palabras clave: Aparato abductor posterior, artroplastia total de cadera, transferencia muscular de glúteo.

\section{ABSTRACT}

Injury to the hip abductor apparatus is a well-characterized clinical entity that conditions pain over the greater trochanter and is accompanied by progressive weakness and claudication. These injuries can occur in patients who underwent total hip replacement or with native hips. Treatment options depend on the location of the injury and the degree of fat-muscle infiltration in both situations. The muscle transfer described by Whiteside from the gluteus maximus to the greater trochanter provides adequate results for pain control and increases strength of the abductor mechanism. In this clinical case we present a patient who underwent a left total hip arthroplasty developed a complete tendon rupture of the gluteus medius, as well as the diagnostic and therapeutic approach of this injury.

Keywords: Posterior abductor apparatus, total hip arthroplasty, gluteal muscle transfer.
* Residente de segundo año de Ortopedia y Traumatología.

‡ Residente de cuarto año de Ortopedia y Traumatología.

$\S$ Cirujano Traumatólogo y Ortopedista con Subespecialidad en Reconstrucción Articular de Cadera y Rodilla.

Centro Médico ABC. México.

Recibido: 04/02/2021. Aceptado: 06/06/2021.

Correspondencia:

Jorge Arturo Barraza-Silva

E-mail: jorge_arturo77@ hotmail.com

\section{INTRODUCCIÓN}

La lesión del aparato abductor de la cadera es una entidad clínica bien caracterizada que condiciona dolor sobre el trocánter mayor, y es acompañado de debilidad y claudicación progresivas. La transferencia descrita por Whiteside del glúteo máximo al trocánter mayor otorga adecuados resultados para el control del dolor y aumento de fuerza del mecanismo abductor. ${ }^{1}$ 
La deficiencia del aparato abductor se debe, por lo general, a una lesión del glúteo medio o a la ruptura del sitio de inserción. Puede originarse en casos con cadera nativa o posterior a una artroplastia total de cadera. El cuadro clínico consiste en marcha de Trendelenburg, claudicación, dolor localizado en la región glútea y trocánter mayor, disminución de la fuerza para la abducción y signo de Trendelenburg. ${ }^{2}$ Su diagnóstico se realiza con la clínica apoyado de ultrasonido o resonancia magnética, siendo la última la más sensible y específica. ${ }^{3,4}$ Existen distintas técnicas quirúrgicas para el tratamiento de esta patología, la reparación directa transósea, en casos crónicos con retracción y sin infiltración grasa se utiliza aloinjerto de tendón de Aquiles y en los casos con infiltración grasa se utiliza la transferencia del glúteo mayor al trocánter mayor. ${ }^{2}$ Estas técnicas ofrecen resultados clínicos adecuados y en este manuscrito se presenta el caso de una mujer de 68 años con ruptura del tendón del glúteo medio posterior a una artroplastia total de cadera izquierda por abordaje lateral directo, así como una revisión de la literatura de esta patología.

\section{REPORTE DE CASO}

Mujer de 68 años con antecedente de coxartrosis izquierda, motivo por el cual fue sometida a artroplastia total de cadera izquierda por abordaje lateral directo y sin otros antecedentes médicos de im-

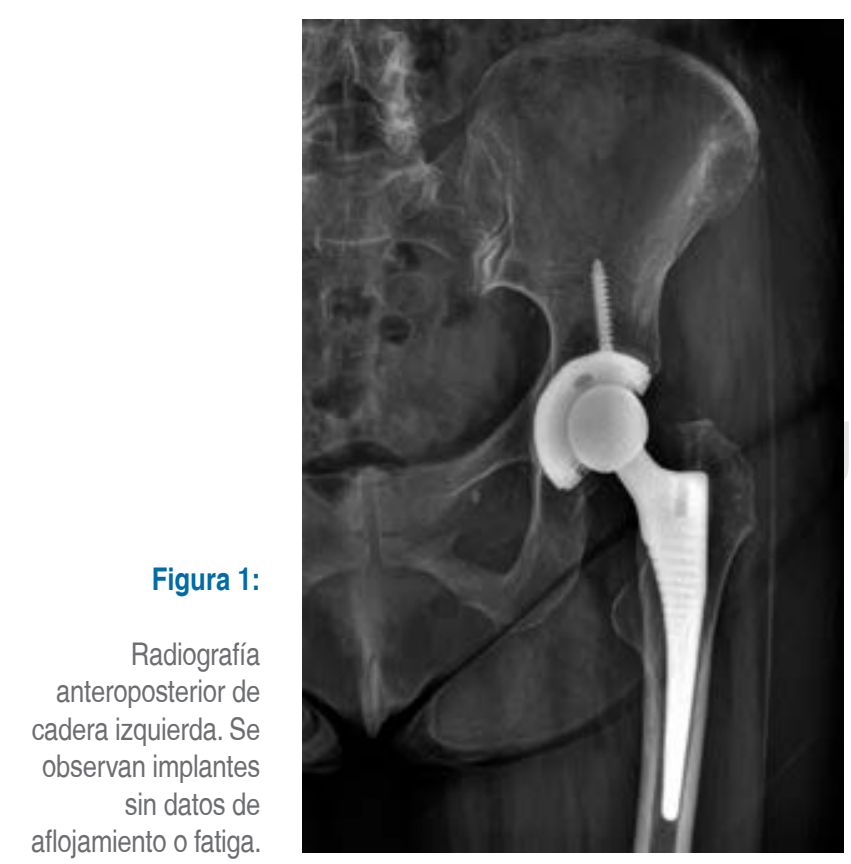

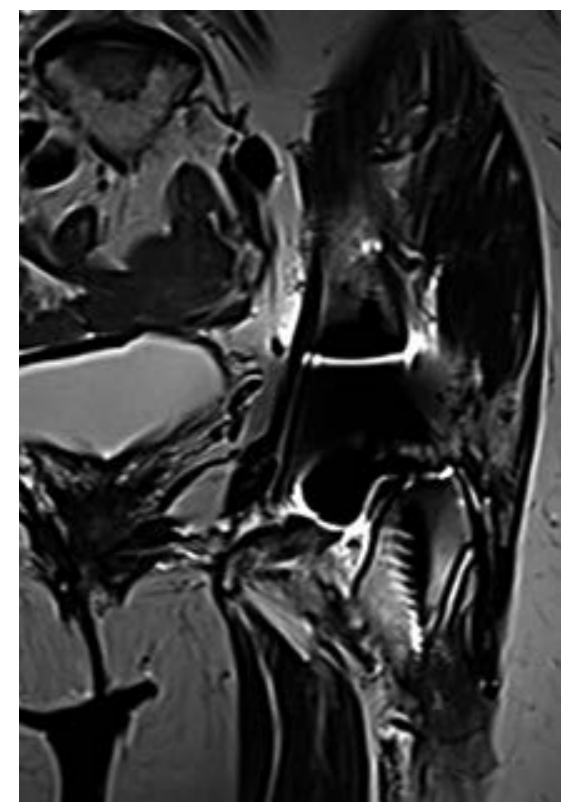

Figura 2:

Corte coronal de resonancia magnética de cadera izquierda en secuencia T1. Se observa la lesión de la inserción del tendón del glúteo medio.

portancia. Es referida al servicio para su evaluación por presentar dolor intenso en región trocantérica, que inició de forma paulatina y progresiva después del procedimiento quirúrgico. Este dolor se irradia hacia la región glútea ipsilateral, es exacerbado con la marcha y atenuado con la ingesta de analgésicos orales. Además del dolor, ha presentado claudicación y debilidad, por lo que ha necesitado de auxiliares de la marcha (bastón) para la deambulación. No ha presentado inestabilidad protésica y a la exploración física se observa marcha en Trendelenburg, rangos de movimiento de cadera limitados a la abducción $20^{\circ}$ por exacerbación de dolor y resto simétricos a contralateral, palpación dolorosa sobre trocánter mayor, signo de Trendelenburg positivo y fuerza para la abducción $3 / 5$ en la escala de Daniels.

Como protocolo diagnóstico, se solicitó una radiografía anteroposterior de pelvis en la cual se observó el estado de los componentes protésicos sin cambios a comparación de la radiografía inicial posterior a la artroplastia total de cadera izquierda, con lo cual se descartó aflojamiento aséptico o fatiga del material (Figura 1). También una resonancia magnética no contrastada en la cual se observó tendinosis insercional del glúteo menor y avulsión > 50\% anterior de la inserción del glúteo medio con infiltración grasa en menor cantidad a las fibras musculares de su vientre muscular (Figura 2).

Con base en los hallazgos de la resonancia magnética, el tiempo de evolución y la clínica de la paciente, logramos categorizar la lesión con el siste- 
ma de clasificación de las lesiones musculares de los abductores de la cadera como una lesión tipo II B con avulsión de $>50 \%$ de la inserción del glúteo medio con un grado de infiltración grasa Goutallier $2 .^{5}$ Estas lesiones no se benefician de tratamiento conservador y deben abordarse quirúrgicamente, ya sea con reparación o con transferencia tendinosa del glúteo mayor. ${ }^{1,5}$

Se optó por una reparación del tendón del glúteo medio con un ancla metálica de $5 \mathrm{~mm}$ de doble sutura, realizando pase tendinoso tipo Krakow (Figura 3). Asimismo, se le realizó la transferencia de la porción anterior del músculo glúteo mayor al trocánter mayor, realizando la tenodesis sobre porción lateral femoral decorticada con tres suturas transóseas anteriores y tres posteriores, utilizando puntos simples con tereftalato de etileno del 1 (Ethibond Johnson \& Johnson ${ }^{\circledR}$ ), dos suturas transóseas distales con extensión proximal tipo Krakow con tereftalato de etileno del 1 (Ethibond Johnson \& Johnson ${ }^{\circledR}$ ), y reforzamiento de transferencia con anclaje a vasto lateral, utilizando puntos en cruz con poliglactina 910 del 2 (Vicryl Johnson \& Johnson ${ }^{\circledR}$ ) (Figura 4).

\section{DISCUSIÓN}

Las lesiones del aparato abductor en pacientes sometidos a artroplastia total de cadera son motivo frecuente de insatisfacción, ya que condicionan dolor, claudicación y limitación funcional. ${ }^{2}$ Cuentan

Figura 3:

Colocación de ancla metálica para reparación de tendón de glúteo medio.

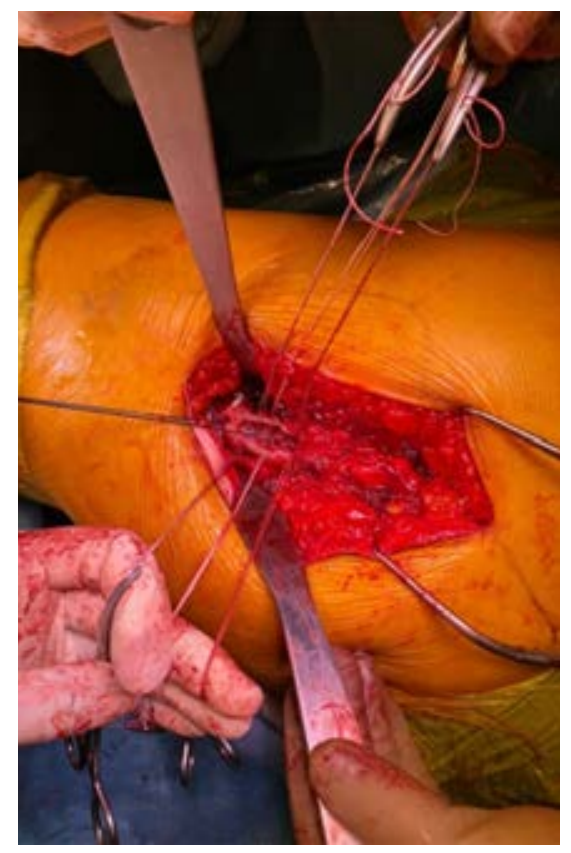

Figura 4:

Decorticación de trocánter mayor y tres suturas transóseas anteriores tres posteriores y una distal para la fijación del tendón del glúteo mayor.

con una prevalencia global de 20 a $22 \%, 6,7$ aunque se ha demostrado una correlación directa con el abordaje utilizado, posterior $0-16 \%$ y anterolateral o lateral 4-20\%. ${ }^{8}$ Lübbeke y colaboradores reportan una prevalencia de $0.7 \%$ de pacientes con necesidad de reparación de los abductores después de una artroplastia total de cadera, utilizando el abordaje lateral directo. ${ }^{9}$

Pueden ocurrir de forma preoperatoria, transoperatoria o postoperatoria ${ }^{2}$ y existen dos principales causas de las lesiones trans y postoperatorias: la lesión directa durante la preparación del canal femoral con subsecuente ruptura tendinosa, y la falla en la reparación de la porción anterior del glúteo medio que se libera del trocánter mayor. ${ }^{9-13}$

El diagnóstico temprano de esta patología es de suma importancia por su implicación en el decremento de la funcionalidad de los casos. El inicio de los síntomas, la ubicación y duración del dolor y los antecedentes traumáticos o luxaciones son datos pivote en los cuales debemos basar nuestra anamnesis y evaluación clínica. A la exploración física hay que buscar claudicación, marcha de Trendelenburg, discrepancia de la longitud de los miembros pélvicos, disminución de la fuerza para la abducción y el signo de Trendelenburg. ${ }^{2}$

La debilidad del mecanismo abductor posterior a una artroplastia total de cadera suele ser mayor en mujeres de edad avanzada que en hombres. ${ }^{6,7,9-13}$ En un metaanálisis publicado en el 2006 por Jolles 
y Bogoch no se encontró diferencia en cuanto a la incidencia de la marcha de Trendelenburg, correlacionándola con el tipo de abordaje utilizado. ${ }^{14}$ Picado y su equipo realizaron electromiografías del glúteo medio de forma preoperatoria y postoperatoria a la artroplastia total de cadera por abordaje lateral directo, encontraron que $42.5 \%$ tenían cambios electromiográficos a las cuatro semanas y $7.5 \%$ a las 24 semanas; de estos últimos, sólo el $2.5 \%$ tenía signo de Trendelenburg positivo. ${ }^{15}$ Svensson y su equipo realizaron un estudio en casos sometidos a artroplastia total de cadera por abordaje transglúteo. La lesión glútea fue reparada con suturas metálicas que podían ser visualizadas por control radiográfico. Encontraron una correlación entre los pacientes que presentaron una separación de más de $2.5 \mathrm{~cm}$ de las suturas y claudicación. ${ }^{16}$

Dentro de los estudios de gabinete que deben ser solicitados, se inicia con una radiografía anteroposterior de pelvis para descartar diagnósticos diferenciales, como alteración en la posición de los implantes y en el offset, así como datos de aflojamiento periprotésico. ${ }^{2}$ García y colaboradores describen el ultrasonido como un método fiable para detectar y medir la extensión de las rupturas de los tendones abductores; sin embargo, continúa siendo operador dependiente. ${ }^{3}$ La artrografía es un estudio poco específico para diagnosticar esta patología ${ }^{2}$ y la resonancia magnética es el estudio más sensible y específico para clasificar roturas de los tendones de los músculos abductores. En esta última, se pueden observar cambios de intensidad en las zonas de lesión y la presencia o no de degeneración grasa muscular. La única desventaja de la resonancia magnética es que puede llegar a sobreestimar el número de lesiones de aparato abductor cuando se correlaciona con la clínica. ${ }^{4}$

Existe un gran espectro de terapias para las lesiones de los tendones del aparato abductor y es que su uso depende del grado de infiltración grasa muscular, la retracción tendinosa y el porcentaje de avulsión tendinosa del trocánter mayor. ${ }^{5}$

Las lesiones que no presentan avulsión del trocánter mayor deben ser tratadas de forma conservadora, en principio por su alta tasa de recuperación y sólo deberán ser intervenidas quirúrgicamente si falla este tratamiento inicial. ${ }^{5}$ Las técnicas utilizadas son la reparación directa transósea o con aloinjerto de tendón de Aquiles en casos que tengan retracción tendinosa. ${ }^{5}$ Lüebbke y colaboradores realizaron reparación del tendón del glúteo medio al trocánter mayor con túneles transóseos y reportan que 50\% de los pacientes presentan mejoría significativa en cuanto al dolor y a la cojera. Sólo el $39 \%$ de los pacientes estuvieron completamente satisfechos con el procedimiento. ${ }^{9}$

Para los casos que cuentan con degeneración grasa muscular y avulsión del trocánter mayor, existe la técnica quirúrgica descrita por Whiteside, que consta de una transferencia tendinosa del glúteo mayor al trocánter mayor. ${ }^{1}$ En su estudio, publicó los resultados funcionales de 11 pacientes con lesión del aparato abductor con signo de Trendelenburg positivo y pérdida de fuerza de la abducción de la cadera por una media de seguimiento de 15 meses. Encontró que nueve pacientes mejoraron significativamente en la fuerza de abducción, presentaron marcha sin claudicación y signo de Trendelenburg negativo. ${ }^{1}$

\section{CONCLUSIÓN}

La evidencia en cuanto al procedimiento de elección para roturas de larga evolución con avulsión del sitio de inserción del glúteo medio aún es escasa, y se deberán realizar estudios comparativos para optar por el mejor tratamiento.

\section{REFERENCIAS}

1. Whiteside LA. Surgical technique: transfer of the anterior portion of the gluteus maximus muscle for abductor deficiency of the hip. Clin Orthop Relat Res. 2012; 470 (2): 503-510.

2. Odak S, Ivory J. Management of abductor mechanism deficiency following total hip replacement. Bone Joint J. 2013; 95-B (3): 343-347.

3. Garcia FL, Picado CH, Nogueira-Barbosa MH. Sonographic evaluation of the abductor mechanism after total hip arthroplasty. J Ultrasound Med. 2010; 29 (3): 465-471.

4. Pfirrmann CW, Notzli HP, Dora C, Hodler J, Zanetti M. Abductor tendons and muscles assessed at MR imaging after total hip arthroplasty in asymptomatic and symptomatic patients. Radiology. 2005; 235 (3): 969-976.

5. Incavo SJ, Harper KD. Open hip abductor tendon repair into a bone trough: improved outcomes for hip abductor tendon avulsion. JBJS Essent Surg Tech. 2020; 10 (2): e0042.

6. Bunker TD, Esler CN, Leach WJ. Rotator-cuff tear of the hip. J Bone Joint Surg Br. 1997; 79 (4): 618-620. doi: 10.1302/0301-620x.79b4.7033.

7. Howell GE, Biggs RE, Bourne RB. Prevalence of abductor mechanism tears of the hips in patients with osteoarthritis. J Arthroplasty. 2001; 16 (1): 121-123.

8. Masonis JL, Bourne RB. Surgical approach, abductor function, and total hip arthroplasty dislocation. Clin Orthop Relat Res. 2002; (405): 46-53.

9. Lübbeke A, Kampfen S, Stern R, Hoffmeyer P. Results of surgical repair of abductor avulsion after primary total hip arthroplasty. J Arthroplasty. 2008; 23 (5): 694-698.

10. Miozzari HH, Dora C, Clark JM, Notzli HP. Late repair of abductor avulsion after the transgluteal approach for hip arthroplasty. J Arthroplasty. 2010; 25 (3): 450-457.e1. 
11. Weber M, Berry DJ. Abductor avulsion after primary total hip arthroplasty. Results of repair. J Arthroplasty. 1997; 12 (2): 202-206.

12. Fehm MN, Huddleston JI, Burke DW, Geller JA, Malchau H. Repair of a deficient abductor mechanism with Achilles tendon allograft after total hip replacement. J Bone Joint Surg Am. 2010; 92 (13): 2305-2311.

13. Khan T, Knowles D. Damage to the superior gluteal nerve during the direct lateral approach to the hip: a cadaveric study. J Arthroplasty. 2007; 22 (8): 1198-1200.
14. Jolles BM, Bogoch ER. Posterior versus lateral surgical approach for total hip arthroplasty in adults with osteoarthritis. Cochrane Database Syst Rev. 2006; (3): CD003828.

15. Picado CH, Garcia FL, Marques W Jr. Damage to the superior gluteal nerve after direct lateral approach to the hip. Clin Orthop Relat Res. 2007; 455: 209-211.

16. Svensson O, Sköld S, Blomgren G. Integrity of the gluteus medius after the transgluteal approach in total hip arthroplasty. J Arthroplasty. 1990; 5 (1): 57-60. 